\title{
FINITE INVARIANT MEASURES ON FLOWS
}

\author{
ROBERT S. Y. WONG
}

(Communicated by Palle E. T. Jorgensen)

\begin{abstract}
We prove that an ergodic flow admits a finite invariant measure if and only if the Kreiger factor whose flow of weights is the flow contains a III $_{1}$ subfactor which is the range of a faithful normal semifinite conditional expectation.
\end{abstract}

\section{INTRODUCTION}

The Murray Von Neumann construction, the flow of weights and the stable range map give 1-1 correspondences between the objects of the categories of ergodic transformations, Kreiger factors and ergodic flows. The proof of the equivalence of these categories has not been completed. However, it is desirable to identify certain properties of objects in one category with some properties in another category. This is like setting up a dictionary for the categories. An example of this is the early work of Connes and Woods on the equivalence of ITPFI factors and the AT-property for flows [2]. Few works have been done in this area since, probably due to the difficulty in proofs or even in guessing the identification. Here we will start with the simple property of a flow admitting a finite invariant measure, and identify it with its corresponding Kreiger factor having a $\mathrm{III}_{1}$ subfactor and an f.n.s. conditional expectation onto it.

Let $\left(F_{t}, \Omega, \nu\right)$ be a flow on a standard measure space. The flow is said to admit an invariant measure if there exists a $\nu^{\prime} \sim \nu$ such that

$$
\nu^{\prime}\left(F_{t}(A)\right)=\nu^{\prime}(A)
$$

for all measurable set $A$ in $\Omega$, and for all $t \in \mathbb{R} . \nu^{\prime}$ is called an invariant measure for the flow.

Proposition 1.1. If an ergodic flow admits an invariant measure, then it is unique up to a scalar multiple.

Proof. Let $\nu_{1}, \nu_{2}$ be two invariant measures for the flow $F_{t}$. We have $\nu_{1} \sim \nu_{2}$, and

$$
\frac{d \nu_{1}}{d \nu_{2}} \circ F_{t}=\frac{d \nu_{1} \circ F_{t}}{d \nu_{2} \circ F_{t}}=\frac{d \nu_{1}}{d \nu_{2}} .
$$

Received by the editors June $28,1990$.

1980 Mathematics Subject Classification (1985 Revision). Primary 46L55. 
So $d \nu_{1} / d \nu_{2}$ is an $F_{t}$ invariant function on $\Omega$, and must be equal to a constant by ergodicity.

By the proposition, the cases that (i) $F_{t}$ does not admit an invariant measure, (ii) $F_{t}$ admits a finite invariant measure, and (iii) $F_{t}$ admits an infinite invariant measure are mutually exclusive.

Theorem 1.2. Let $M$ be a $I I I_{0}$ Kreiger factor. The flow of weights of $M$ admits a finite invariant measure if and only if there exists a $I I I_{1}$ subfactor of $M$ which is the range of a faithful normal conditional expectation.

Proof. (If part) Let $\left(F_{t}, \Omega, \nu\right)$ be the flow of weights of $M$. By changing to a equivalent measure if necessary, we can assume that $\nu$ is the invariant measure, and that $\nu(\Omega)=1$. Let $\mathbf{X}_{3}=\prod_{1}^{\infty} \mathbf{Z}_{3}$, where $\mathbf{Z}_{3}$ is the cyclic group of order 3 , and let $p=\prod_{1}^{\infty}\left(\frac{1}{2}, \frac{1}{3}, \frac{1}{6}\right)$ be the product measure of the constant measure $\left(\frac{1}{2}, \frac{1}{3}, \frac{1}{6}\right)$ on $\mathrm{Z}_{3}$. Then the odometer transformation $T$ is of type $\mathrm{III}_{1}$ and the system $\left(S, \mathbf{X}_{3} \times \Omega, p \times \nu\right)$ where

$$
S(x, \omega)=\left(T x, F_{a(x)}(\omega)\right) \quad(x, \omega) \in \Omega,
$$

and

$$
a(x)=\log \frac{d p \circ T}{d p}(x)
$$

has the flow as its stable range (see for example [4]). By Krieger's theorem,

$$
M \simeq W^{*}\left(S, \mathbf{X}_{3} \times \Omega, p \times \nu\right) .
$$

Let $M_{1}$ be the cross product $W^{*}\left(T, \mathbf{X}_{3}, p\right) . M_{1}$ is a $I I I_{1}$ factor. We shall construct an embedding $i$ of $M_{1}$ into $M$ and a faithful normal conditional expectation $E: M \rightarrow i\left(M_{1}\right)$. For convenience, we shall identify the spaces $L_{2}(X \times \mathbb{Z}), \ell^{2}\left(\mathbb{Z}, L_{2}(X)\right), L^{2}(X) \otimes \ell^{2}(\mathbb{Z})$ etc. in the canonical way.

The map $i: B\left(L^{2}\left(\mathbf{X}_{3} \times \mathbb{Z}\right)\right) \rightarrow B\left(L^{2}\left(\mathbf{X}_{3} \times \mathbb{Z}\right)\right) \otimes B\left(L^{2}(\Omega)\right) \simeq B\left(L^{2}\left(\mathbf{X}_{3} \times \Omega \times \mathbb{Z}\right)\right)$ given by

$$
i(x)=x \otimes 1
$$

is a *-monomorphism. $M_{1}$ is generated by elements of the form $\pi_{T}(a), a \in$ $L^{\infty}\left(\mathbf{X}_{3}\right)$, and a single unitary $U_{T}$. For such a $\pi_{T}(a)$ and $\xi \in L^{2}\left(\mathbf{X}_{3} \times \Omega \times \mathbb{Z}\right)$ we have

$i\left(\pi_{T}(a)\right)(\xi)(m)=\left(\pi_{T}(a) \otimes 1\right)(\xi)(m)=\left(a \circ T^{-m} \otimes 1_{\Omega}\right) \xi(m)=\left[\pi_{S}\left(a \otimes 1_{\Omega}\right) \xi\right](m)$

for all $m \in \mathbb{Z}$. Also

$$
i\left(U_{T}\right) \xi(m)=\left(U_{T} \otimes 1\right) \xi(m)=\xi(m-1)=\left(U_{S} \xi\right)(m) .
$$

Hence

$$
i\left(\pi_{T}(a)\right)=\pi_{S}(a \otimes 1)
$$

and

$$
i\left(U_{T}\right)=U_{S} \text {. }
$$

So $i$ embeds $M_{1}$ into $M$ unitally. Let $1_{\Omega}$ denote the constant function 1 on $\Omega$. Since $\nu$ is finite, $1_{\Omega} \in L^{2}(\Omega)$. There is an isometry $v: L^{2}\left(\mathbf{X}_{3} \times \mathbb{Z}\right) \rightarrow$ $L^{2}\left(\mathbf{X}_{3} \times \mathbb{Z}\right) \otimes L^{2}(\Omega) \simeq L^{2}\left(\mathbf{X}_{3} \times \Omega \times \mathbb{Z}\right)$ given by

$$
v \xi=\xi \otimes 1_{\Omega}
$$


The conditional expectation is given by

$$
E(x)=i\left(v^{*} x v\right), \quad x \in M .
$$

We show that $E$ is indeed a conditional expectation. For $x \in M_{1}$, and $\xi \in$ $L^{2}\left(\mathbf{X}_{3} \times \mathbb{Z}\right)$ we have

$$
v^{*} i(x) v(\xi)=v^{*}(x \otimes 1)\left(\xi \otimes 1_{\Omega}\right)=\int x(\xi) \otimes 1_{\Omega} d \nu=x(\xi) .
$$

Hence $E(x)=x$ for all $x \in i\left(M_{1}\right)$. In particular $E\left(U_{S}\right)=i\left(U_{T}\right)=U_{S}$. Also for $a \in L^{\infty}\left(\mathbf{X}_{3} \times \Omega\right)$ and $\xi \in L^{2}\left(\mathbf{X}_{3} \times \mathbb{Z}\right)$ we have

$$
\begin{aligned}
\left(v^{*} \pi_{S}(a) v\right) \xi(x, m) & =\int\left(\pi_{S}(a) v \xi\right)(x, m, \omega) d \nu(\omega) \\
& =\int a \circ S^{-m}(x, \omega) \xi(x, m) d \nu(\omega) \\
& =\left(\int a(x, \omega) d \nu(\omega)\right) \circ T^{-m} \xi(x, m) \\
& =\pi_{T}(b) \xi(x, m) \text { for all } m \in \mathbb{Z} \text {, a.e. } x \in \mathbf{X}_{3}
\end{aligned}
$$

where $b \in L^{2}\left(\mathbf{X}_{3}\right), b(x)=\int a(x, \omega) d \nu(\omega)$. Hence $E\left(\pi_{S}(a)\right)=i \pi_{T}(b) \epsilon$ $i\left(M_{1}\right)$. Using the normality of $E$, we see that $E$ is a projection from $M$ onto $M_{1}$. From

$$
\|E(x)\|=\left\|v^{*} x v\right\| \leq\|x\|
$$

and

$$
E(1)=v^{*} v \otimes 1=1,
$$

we conclude that $E$ is a projection of norm 1 , and hence a conditional expectation. To show that $E$ is faithful, let $A=\pi_{S}\left(L^{\infty}\left(\mathbf{X}_{3} \times \Omega\right)\right)$ and $F: M \rightarrow A$ be the canonical conditional expectation; i.e., if $x$ has the representation

$$
x=\sum_{n \in \mathbb{Z}} \pi_{S}\left(a_{n}\right) U^{n}, \quad a_{n} \in L^{\infty}\left(\mathbf{X}_{3} \times \Omega\right),
$$

then $F(x)=\pi_{S}\left(a_{0}\right) . \quad F$ is normal and faithful. By a calculation similar to the one above, we get $E\left(\pi_{S}(a) U^{n}\right)=E\left(\pi_{S}(a)\right) U^{n}$ for all $a \in L^{\infty}\left(\mathbf{X}_{3} \times \Omega\right)$ and $n \in \mathbb{Z}$. Using the normality of $E$ and $F$, we see that $E \circ F=F \circ E$. Let $E\left(x x^{*}\right)=0$ for some $x \in M$. Then $E F\left(x x^{*}\right)=F E\left(x x^{*}\right)=0$. Since $E$ is obviously faithful on $A, F\left(x x^{*}\right)=0$ and hence $x=0$.

(Only if part) Now we prove the converse of the theorem. As in [2], we let

$$
\tilde{M}=M \otimes F_{\infty}, \quad \tilde{M}_{1}=M_{1} \otimes F_{\infty}, \quad \tilde{E}=E \otimes 1, \quad \theta_{s}=\operatorname{Ad}\left(1 \otimes V_{s}\right),
$$

where $F_{\infty}=B\left(L^{2}(\mathbb{R})\right)$ and $\left(V_{s} \xi\right)(t)=\xi(t-s)$. We have for $x \otimes y \in M \otimes F_{\infty}$,

$$
\begin{aligned}
\tilde{E} \circ \theta_{s}(x \otimes y) & =\tilde{E}\left(x \otimes \operatorname{Ad}\left(V_{s}\right)(y)\right)=E(x) \otimes \operatorname{Ad} V_{s}(y) \\
& =\left(1 \otimes \operatorname{Ad} V_{s}\right)(E(x) \otimes y)=\theta_{s} \tilde{E}(x \otimes y) .
\end{aligned}
$$

Hence $\tilde{E}$ is a faithful normal conditional expectation which commutes with $\theta_{s}$. Let $\phi_{1}$ be a faithful normal semifinite weight on $M_{1}$ and let $\phi=\phi_{1} \circ E$. We write

$$
\tilde{\phi}=\phi \otimes \omega, \quad \tilde{\phi}_{1}=\phi_{1} \otimes \omega, \quad N=\tilde{M}_{\tilde{\phi}}, \quad N_{1}=\tilde{M}_{1 \tilde{\phi}_{1}} .
$$


By Takesaki's theorem, $\sigma_{t}^{\phi} \mid M_{1}=\sigma_{t}^{\tilde{\phi}_{1}}$, so $N_{1}$ is a unital subalgebra of $N$. We also have

$$
E \circ \sigma_{t}^{\phi}=\sigma_{t}^{\phi_{1}} \circ E
$$

Hence

$$
\tilde{E} \circ \sigma_{t}^{\tilde{\phi}}=(E \otimes 1) \circ\left(\sigma_{t}^{\phi} \otimes 1\right)=\left(\sigma_{t}^{\phi_{1}} \circ E\right) \otimes 1=\sigma_{t}^{\tilde{\phi}_{1}} \circ \tilde{E},
$$

so that $\tilde{E}(N)=N_{1}$. Now we again let $E$ denote the restriction of $\tilde{E}$ to $C=\mathbf{Z}(N)$. Then $E$ commutes with $\theta_{s}$, and for $f \in C, y \in N_{1}$, we have

$$
y E(f)=E(y f)=E(f y)=E(f) y .
$$

So $E(f) \in \mathbf{Z}\left(N_{1}\right)=\mathbb{C} 1$. Hence $E$ is a faithful normal functional which is invariant under the flow of weights of $M$, which is $\theta_{s} \mid C$. This means that $E$ is the finite invariant measure for the flow.

Remark. In the proof of the "only if" part of the theorem (i.e. if $M$ admits a III $_{1}$ subfactor which is the range of a faithful normal conditional expectation, then the flow of weights of $M$ admits a finite invariant measure), we did not use the fact that $M$ is a Kreiger factor, nor even that it is a factor. Hence half of the theorem is true for all $\mathrm{III}_{0}$ von Neumann algebras $M$.

The above result for finite invariant measure suggests that a similar result should be valid in the case of infinite invariant measures. Naturally we should consider the notion of an "unbounded" conditional expectation, analogous to the extension of a state to a weight. This has been considered in [3], where operator valued weight is defined. Using the machinery from [1], it is possible to prove that, if the flow of weights of a Kreiger factor $M$ admits an infinite invariant measure, then there exists a normal faithful semifinite operator valued weight from $M$ onto a subfactor of type III $_{1}$. However, the converse, though possibly true, cannot be proved by the same method as above. The difficulty is due to the fact that the restriction of a semifinite operator valued weight to a subalgebra need not be semifinite.

\section{REFERENCES}

1. A. Connes, Sur la theorie non commutative de l'integration, Lecture Notes in Math., vol. 725, Springer-Verlag, 1979, pp. 19-143.

2. A. Connes and E. J. Woods, Approximate transitive flows and ITPFI factors, Ergodic Theory Dynamical Systems 5 (1985), 203-236.

3. U. Haagerup, Operator valued weights. I, J. Funct. Anal. 32 (1979), 176-206; II, ibid. 33 (1979), 339-361.

4. T. Hamachi and M. Osikawa, Ergodic groups of automorphisms and Krieger's theorems, Sem. Math. Sci. No.3, Keio University, 1981.

5. S. Stratila, Modular theory in operator algebras, Abascus Press, 1981.

6. M. Takesaki, Theory of operator algebras 1, Springer-Verlag, 1979.

School of Mathematics, University of New South Wales, Kensington, N. S. W., Australia 